\title{
Automedicação entre estudantes de enfermagem em uma universidade privada no sul de Minas Gerais
}

\author{
Self-medication among nursing students at a private university in southern Minas Gerais \\ La automedicación en estudiantes de enfermería de una universidad privada del sur de Minas \\ Gerais
}

Recebido: 13/06/2021 | Revisado: 24/06/2021 | Aceito: 26/06/2021 | Publicado: 11/07/2021

\author{
Cibele Dutra Pinto \\ ORCID: https://orcid.org/0000-0002-7494-7171 \\ Universidade José do Rosário Vellano, Brasil \\ E-mail: cibele.pinto@aluno.unifenas.br \\ Natália de Oliveira \\ ORCID: https://orcid.org/0000-0002-1694-7536 \\ Universidade José do Rosário Vellano, Brasil \\ E-mail: natalia_oliveira95@hotmail.com \\ Roberta Bessa Veloso Silva \\ ORCID: https://orcid.org/0000-0003-4794-5872 \\ Universidade José do Rosário Vellano, Brasil \\ E-mail: bessaveloso@yahoo.com.br \\ Cláudio Daniel Cerdeira \\ ORCID: https://orcid.org/0000-0002-7242-8028 \\ Universidade José do Rosário Vellano, Brasil \\ E-mail: daniel.cerdeira.84@gmail.com \\ José Antonio Dias Garcia \\ ORCID: https://orcid.org/0000-0002-4024-3045 \\ Universidade José do Rosário Vellano, Brasil \\ E-mail: jadiasgarcia@gmail.com \\ Gérsika Bitencourt Santos Barros \\ ORCID: https://orcid.org/0000-0003-0849-2786 \\ Universidade José do Rosário Vellano, Brasil \\ E-mail: gersikasantos@unifenas.br
}

\begin{abstract}
Resumo
Devido à facilidade para a aquisição de algumas classes de medicamentos, sem a necessidade de prescrição médica e, sob influência de estímulos nocivos dos meios de comunicação, a automedicação tornou-se uma conduta corriqueira que traz graves riscos à saúde e qualidade de vida da população. Este estudo analisou a frequência de automedicação entre alunos do curso de Enfermagem em uma universidade no sul de Minas Gerais, Brasil. Um questionário semiestruturado sobre automedicação foi aplicado aos discentes, em 2018. Entre 110 entrevistados, de 17 a 46 anos de idade, houve predomínio do sexo feminino $(78,18 \%)$ na faixa etária entre 20-30 anos. Houve alta frequência de estudantes que relataram a prática de automedicação $(94,5 \%)$, inclusive para medicamentos de uso obrigatório de receitas (venda apenas sob prescrição) e, sendo este hábito, mais comum entre os alunos dos últimos períodos do curso. O reaproveitamento de receitas e medicamentos de tratamentos anteriores foi também comum. Os problemas de saúde mais relatados pelos entrevistados que levaram à automedicação foram: dor de cabeça $(87,20 \%)$, gripes e resfriados $(82,7 \%)$, febre $(55,4 \%)$, alergias $(52,7 \%)$ e infecções/inflamações $(46,3 \%)$. As classes de medicamentos mais usadas foram: analgésicos/antipiréticos $(86,3 \%)$, antigripais/medicamentos para tratar resfriado $(84,5 \%)$, xaropes $(82,7 \%)$, anti-inflamatórios $(74,5 \%)$ e antialérgicos/anti-histamínicos $(56,3 \%)$. Conclui-se que é alta a frequência de automedicação entre acadêmicos de enfermagem, sendo mais comum este hábito entre os alunos de períodos mais adiantados do curso.
\end{abstract}

Palavras-chave: Automedicação; Promoção de saúde; Medicamentos.

\begin{abstract}
Due to the easy way for the people to acquire some classes of medicines, without the need for a medical prescription and, under the influence of harmful stimuli from the media, self-medication has become a common conduct that poses serious risks to the population's health and quality of life. This study analyzed the frequency of self-medication among nursing students at a university in southern Minas Gerais, Brazil. A semi-structured questionnaire on self-medication was applied to students in 2018. Among 110 respondents, from 17 to 46 years of age, there was a predominance of
\end{abstract}


females $(78.18 \%)$ in the age group between 20-30 years. There was a high frequency of students who reported the practice of self-medication (94.5\%), including for prescription medicines and, being this habit, more common among students from the last periods of the course. The reuse of prescriptions and medications from previous treatments was also common. The health problems most reported by the interviewees that led to self-medication were as following: headache $(87.20 \%)$, flu and colds $(82.7 \%)$, fever $(55.4 \%)$, allergies $(52.7 \%)$ and infections/inflammations $(46.3 \%)$. The most used medicine classes were as following: analgesics/antipyretics $(86.3 \%)$, flu/medicines to treat colds $(84.5 \%)$, syrups $(82.7 \%)$, anti-inflammatories $(74.5 \%)$ and antiallergics/antihistamines $(56.3 \%)$. It is concluded that the frequency of self-medication among nursing students is high, with this habit being more common among students from the last periods of the course.

Keywords: Self-medication; Health promotion; Medicines.

\section{Resumen}

Debido a la facilidad para adquirir algunas clases de fármacos, sin necesidad de prescripción médica y bajo la influencia de estímulos nocivos de los medios de comunicación, la automedicación se ha convertido en una conducta habitual que plantea graves riesgos para la salud y calidad de vida de la población. Este estudio analizó la frecuencia de automedicación entre estudiantes de enfermería de una universidad del sur de Minas Gerais, Brasil. En 2018 se aplicó un cuestionario semiestructurado sobre automedicación a los estudiantes. Entre 110 estudiantes analizados, de 17 a 46 años, hubo predominio del sexo femenino (78,18\%) en el grupo de edad entre 20-30 años. Hubo una alta frecuencia de estudiantes que refirieron la práctica de la automedicación (94,5\%), incluso para medicamentos de prescripción y, siendo este hábito, más común entre los estudiantes de los últimos periodos del curso. La reutilización de recetas y medicamentos de tratamientos anteriores también fue común. Los problemas de salud más reportados por los entrevistados que llevaron a la automedicación fueron: dolor de cabeza (87,20\%), gripes y resfriados $(82,7 \%)$, fiebre $(55,4 \%)$, alergias $(52,7 \%)$ e infecciones/inflamaciones $(46,3 \%)$. Las clases de medicamentos más utilizadas fueron: analgésicos/antipiréticos $(86,3 \%)$, antigripales/medicamentos para el resfriado $(84,5 \%)$, jarabes $(82,7 \%)$, antiinflamatorios $(74,5 \%)$ y antialérgicos/antihistamínicos $(56,3 \%)$. Se concluye que la frecuencia de automedicación entre los estudiantes de enfermería es alta, siendo este hábito más común entre los estudiantes de los últimos periodos del curso.

Palabras clave: Automedicación; Promoción de la salud; Medicamentos.

\section{Introdução}

O termo automedicação designa o uso de medicamento sem prescrição médica visando o tratamento de doenças, aliviar sintomas ou até mesmo para promover saúde. O termo estende-se também a automedicação orientada, referindo-se à reutilização de receitas antigas que não são emitidas para medicamentos de uso contínuo (Vilarino et al., 1998).

Alguns fatores contribuem com o cada vez mais crescente hábito de automedicação entre a população brasileira, sendo que, os veículos de comunicação, através de anúncios chamativos e estratégias de marketing, as vezes envolvendo profissionais de saúde, estimulam o consumo indiscriminado e desenfreado de medicamentos sem a prescrição médica, fazendo com que haja grande disponibilidade do produto no mercado, atualmente também alavancado pelas vendas na internet e propagandas em redes sociais. Portanto, estes fatores deletérios potencialmente trazem graves riscos à saúde da população, causando um sério problema de saúde pública no Brasil (Nascimento, 2009; Lyra et al., 2010; Domingues et al., 2017; Alhaddad, 2018; Azevedo et al., 2019; Gimenes et al., 2019).

A automedicação gera riscos, tais como: o mascaramento do diagnóstico da doença; resistência medicamentosa devido ao uso irracional e inadequado (principalmente como visto para os antimicrobianos); potenciais interações medicamentosas; alergias, intoxicação e até mesmo morte (Daniely et al., 2017; Freitas \& Melo, 2018). Segundo a Organização Mundial da Saúde (OMS), grande parte dos óbitos no Brasil são provocados por intoxicação medicamentosa, e em média, aproximadamente $20 \%$ dos orçamentos hospitalares são reservados ao tratamento de complicações causadas pelo uso irracional de medicamentos advindos da automedicação (Pereira et al., 2008).

Baseado no exposto, uma vez que a automedicação se tornou uma conduta rotineira entre a população e isto traz graves riscos à saúde, são necessários estudos que visem conhecer a realidade de certos grupos populacionais quanto à este hábito deletério, visando a elaboração de orientações e estratégias de combate à automedicação (Sousa et al., 2008). Portanto, 
este estudo analisou a frequência de automedicação entre alunos do curso de Enfermagem em uma universidade privada no sul de Minas Gerais.

\section{Metodologia}

Este estudo é de caráter transversal e observacional, com abordagem de natureza quantitativa, de base primária. A amostra foi composta por estudantes dos $1^{\circ}, 3^{\circ}, 5^{\circ}$ e $7^{\circ}$ períodos do curso de Enfermagem de uma universidade privada localizada no sul do estado de Minas Gerais, Brasil. Um questionário semiestruturado foi aplicado durante o segundo semestre de 2018, abordando questões sobre o status demográfico, condições de saúde e uso de medicamentos. Como critérios de inclusão, todos os alunos matriculados no referido curso e nos períodos supracitados, foram inclusos, após concordarem em participar do estudo mediante esclarecimento e assinatura do termo de consentimento livre e esclarecido (TCLE).

Quanto a coleta dos dados, os entrevistadores foram previamente calibrados e foi ressaltado junto aos participantes voluntários possíveis vieses de memória e o período recordatório foi esclarecido para cada variável. Para a análise dos dados utilizou-se o teste de qui-quadrado $\left(\chi_{2}\right)$, ao nível nominal de 5\% de significância para verificar a independência entre as variáveis de interesse. O teste exato de Fisher foi aplicado nos casos em que a frequência absoluta das células da tabela de contingência foi menor ou igual a 5. A análise estatística foi realizada no software $\mathrm{R}^{\circledR}$ (R Core Team, 2020). O intervalo de confiança de $95 \%$ ( IC $_{(95 \%)}$ ) foi projetado utilizando o software BioEstat ${ }^{\circledR} 5.0$ (Belém, Pará, Brasil, 2007). O presente estudo foi desenvolvido após aprovação pelo Comitê de Ética em pesquisa envolvendo seres humanos da Universidade José do Rosário Vellano - UNIFENAS (protocolo de aprovação nº 3.216.696).

\section{Resultados}

A Tabela 1 apresenta os dados demográficos, condições de saúde e dados sobre o uso de medicamentos, na amostra de 110 alunos do curso de enfermagem de uma Universidade privada no Sul de Minas Gerais, onde verifica-se maior frequência de acadêmicas, 86 (78,18\%), com distribuição variada ao longo dos períodos, faixa etária predominantemente entre 20-30 anos. Como visto na Tabela 2, houve diferença significativa entre a faixa etária e o período cursado $(p<0,01)$.

Ainda na Tabela 1, em relação aos problemas de saúde apresentados, a maioria afirmou não ter nenhuma intercorrência com sua saúde (84\%). Por outro lado, na amostra total avaliada, a maioria (94,5\%) já comprou medicamento fazendo tal compra por automedicação. Desses, sobre a finalidade do medicamento, a maioria dos entrevistados $(68,10 \%)$ relatou ser para uso próprio e, $80 \%$, já esqueceu ou perdeu a receita no momento da compra. Ainda, quando necessário, $79 \%$ dos estudantes já pediram algum aconselhamento para o farmacêutico ou balconista responsável na farmácia.

Analisando-se a relação entre outras duas variáveis que houve associação significativa (Tabela 2), "baseou-se em receitas anteriores versus período", das 64 (58,18\%) acadêmicas que relataram terem se baseado em receitas antigas, 14 $(21,87 \%)$ estavam no primeiro período, 12 (18,75\%), no terceiro período, 14 (21,87\%), no quinto período e 24 (37,5\%), sétimo período. Dos 46 que disseram que nunca se baseou em receitas anteriores, 21 (41,82\%), se encontravam no primeiro período, 5 (10,89\%), no terceiro período, 14 (30,43\%), quinto período e 6 (13,04\%), no sétimo período. No primeiro período verificou-se que a frequência de acadêmicos que não se basearam em receitas antigas foi quase 2 vezes maior que àqueles que já o fizeram. Já no quinto período houve maior frequência de uso, sendo 1,7 vezes maior do que os que relataram negativamente. No quinto período, o resultado inverteu, sendo a frequência de quem já se baseou em receitas antigas, 1,4 vezes menor se comparado aos que marcaram "não". Deve-se destacar, no sétimo período, que a frequência dos que se basearam em receitas antigas foi quase 3 vezes maior do que os que disseram não ter usado. 
Sobre os motivos para se medicar ou doenças que o acadêmico relatou ter (Figura 1), o mais mencionado foi a dor de cabeça $(87,20 \%)$, seguido pelo resfriado $(82,70 \%)$ e febre $(55,40 \%)$. Os motivos menos relatados foram: doenças pulmonares $(2,70 \%)$ e outras doenças de cabeça e pescoço (3,60\%). Ainda na Figura 1, no que diz respeito a última consulta médica que o estudante realizou, a maioria dos entrevistados (25,40\%), menciona não lembrar da data da última consulta médica e $24,50 \%$ afirmam que sua última consulta foi de 1 a 3 meses, 20\% como sendo há mais de 3 meses e 15,40\% mais de 1 semana, empatando com as consultas realizadas entre 1 semana e 1 mês. Com relação ao reaproveitamento de medicamentos usados em tratamentos anteriores, $72 \%$ dos entrevistados disseram que sim, reaproveitam medicamentos utilizados anteriormente.

Tabela 1 - Dados demográficos e de saúde/uso de medicamentos na amostra de alunos do curso de enfermagem de uma Universidade privada no Sul de Minas Gerais, 2018.

\begin{tabular}{|c|c|c|c|}
\hline \multirow[t]{2}{*}{ Variáveis } & & \multicolumn{2}{|c|}{ Resultados entre os 110 entrevistados } \\
\hline & & $n(\%)$ & IC95\% \\
\hline \multirow[t]{2}{*}{ Sexo } & Feminino & $86(78,18)$ & $70,5-85,9$ \\
\hline & Masculino & $24(21,82)$ & $14,1-29,5$ \\
\hline \multirow[t]{3}{*}{ Faixa etária (anos) } & $17-20$ & $40(36,30)$ & $27,4-45,4$ \\
\hline & $21-30$ & $59(53,60)$ & $44,3-63$ \\
\hline & $31-46$ & $11(10,00)$ & $4,4-15,6$ \\
\hline \multirow[t]{24}{*}{ Período do curso } & $1^{\mathrm{o}}$ & & \\
\hline & Feminino & $26(23,6)$ & $15,7-31,6$ \\
\hline & Masculino & $9(8,2)$ & $3,1-13,3$ \\
\hline & $17-20$ anos & $23(21)$ & $13,3-28.5$ \\
\hline & $21-30$ anos & $11(10)$ & $4,4-15,6$ \\
\hline & $31-46$ anos & $1(0,9)$ & ---- \\
\hline & $3^{\circ}$ & & \\
\hline & Feminino & $15(13,7)$ & $7,2-20$ \\
\hline & Masculino & $2(1,9)$ & ---- \\
\hline & $17-20$ anos & $9(8,2)$ & $3,1-13,3$ \\
\hline & $21-30$ anos & $6(5,5)$ & $1,2-9,7$ \\
\hline & $31-46$ anos & $2(1,8)$ & --- \\
\hline & $5^{\circ}$ & & \\
\hline & Feminino & $20(18,2)$ & $11-25,4$ \\
\hline & Masculino & $8(7,3)$ & $2,4-12,1$ \\
\hline & $17-20$ anos & $7(6,4)$ & $1,8-10,9$ \\
\hline & $21-30$ anos & $17(15,4)$ & $8,7-22,2$ \\
\hline & $31-46$ anos & $4(3,7)$ & --- \\
\hline & $7^{\circ}$ & & \\
\hline & Feminino & $25(22,7)$ & $14,9-30,6$ \\
\hline & Masculino & $5(4,5)$ & ---- \\
\hline & $17-20$ anos & $1(0,9)$ & ---- \\
\hline & $21-30$ anos & $25(22,7)$ & $14,9-30,6$ \\
\hline & $31-46$ anos & $4(3,7)$ & --- \\
\hline \multirow[t]{2}{*}{ Problema de saúde } & Sim & $18(16)$ & $9,5-23,3$ \\
\hline & Não & $92(84)$ & $76,7-90,5$ \\
\hline Automedicação & Sim & $104(94,5)$ & $90,3-98,8$ \\
\hline \multirow[t]{8}{*}{ (Compra sem receita) } & $1^{\circ}$ período & $33(30)$ & $21,4-38,6$ \\
\hline & $3^{\circ}$ período & $17(15,4)$ & $8,7-22,2$ \\
\hline & $5^{\circ}$ período & $26(23,7)$ & $15,7-31,6$ \\
\hline & $7^{\circ}$ período & $28(25,4)$ & $17,3-33,6$ \\
\hline & Não & $6(5,5)$ & $1,2-9,7$ \\
\hline & $1^{\circ}$ período & $2(1,8)$ & ---- \\
\hline & $3^{\circ}$ período & $0(0)$ & ---- \\
\hline & $5^{\circ}$ período & $2(1,8)$ & ---- \\
\hline
\end{tabular}




\begin{tabular}{|c|c|c|c|}
\hline & $7^{\circ}$ período & $2(1,8)$ & ---- \\
\hline $\begin{array}{l}\text { Medicamento requer "apresentação } \\
\text { obrigatória" de receita }\end{array}$ & $\begin{array}{l}\text { Sim } \\
\text { Não }\end{array}$ & $\begin{array}{l}35(31,8) \\
69(62,7)\end{array}$ & $\begin{array}{l}23,1-40,5 \\
53,7-71,8\end{array}$ \\
\hline $\begin{array}{l}\text { Tempo de uso do medicamento } \\
\text { (dias) }\end{array}$ & $\begin{array}{l}01 \\
02 \\
03-05 \\
>05\end{array}$ & $\begin{array}{c}8(7,2) \\
16(14,5) \\
72(65,4) \\
54(49)\end{array}$ & $\begin{array}{c}2,4-12,1 \\
8-21,1 \\
56,6-74,3 \\
39,7-58,4\end{array}$ \\
\hline Seguiu instruções da bula & $\begin{array}{l}\text { Sim } \\
\text { Não }\end{array}$ & $\begin{array}{l}56(51) \\
54(49)\end{array}$ & $\begin{array}{l}41,6-60,3 \\
39,7-58,4\end{array}$ \\
\hline Reaproveitamento de receitas & $\begin{array}{l}\text { Sim } \\
\text { Não }\end{array}$ & $\begin{array}{l}64(58,10) \\
46(41,90)\end{array}$ & $\begin{array}{l}49-67,4 \\
32,6-51\end{array}$ \\
\hline Finalidade do medicamento & $\begin{array}{l}\text { Uso próprio } \\
\mathrm{P} / \text { familiar } \\
\text { Ambos } \\
\text { Outro }\end{array}$ & $\begin{array}{c}75(68,10) \\
10(9) \\
30(27,20) \\
2(1,8)\end{array}$ & $\begin{array}{c}59,5-76,9 \\
3,7-14,5 \\
18,9-35,6 \\
----\end{array}$ \\
\hline Esquecimento ou perdeu a receita & $\begin{array}{l}\text { Sim } \\
\text { Não }\end{array}$ & $\begin{array}{l}22(20) \\
88(80)\end{array}$ & $\begin{array}{l}12,5-27,5 \\
72,5-87,5\end{array}$ \\
\hline Aconselhamento com farmacêutico & $\begin{array}{l}\text { Sim } \\
\text { Não }\end{array}$ & $\begin{array}{l}87(79) \\
23(21)\end{array}$ & $\begin{array}{l}71,5-86,7 \\
13,3-28.5\end{array}$ \\
\hline $\begin{array}{l}\text { Já recebeu conselhos não solicitados } \\
\text { na farmácia }\end{array}$ & $\begin{array}{l}\text { Sim } \\
\text { Não }\end{array}$ & $\begin{array}{l}64(58,10) \\
46(41,90)\end{array}$ & $\begin{array}{l}49-67,4 \\
32,6-51\end{array}$ \\
\hline
\end{tabular}

Fonte: Autores.

Tabela 2 - Valor-p resultante do teste de independência para as variáveis analisadas, comparando-se período cursado vs uso de medicamentos ou outras variáveis demográficas,

\begin{tabular}{|c|c|}
\hline Variáveis & Valor-p \\
\hline Sexo vs período & $0,4948 n s$ \\
\hline Faixa etária vs período & $<0,01^{* * *}$ \\
\hline Depressão vs período & $0,0752 \mathrm{~ns}$ \\
\hline Medicamento sem receita $v s$ período & $0,8889 n s$ \\
\hline Uso próprio vs período & $0,1518 n s$ \\
\hline Esqueceu ou perdeu a receita $v s$ período & $0,3896 n s$ \\
\hline Aconselhou-se com o farmacêutico ou balconista vs período & $0,1332 n s$ \\
\hline Recebeu conselhos na farmácia $v s$ período & $0,0623 n s$ \\
\hline Aconselhou-se com terceiros vs período & $0,1160 n s$ \\
\hline Aconselhou-se com quem (caso afirmativa a questão anterior) vs período & $0,7584 n s$ \\
\hline Baseou-se em receitas antigas vs período & $0,0059^{* *}$ \\
\hline De quem eram as receitas antigas $v s$ período & $0,3982 n s$ \\
\hline Medicamento com receita $v s$ período & $0,5465 n s$ \\
\hline Número de princípios ativos vs período & $0,7168 n s$ \\
\hline Quais medicamentos se automedicou $v s$ período & $0,9999 n s$ \\
\hline Tempo de uso da medicação vs período & $0,0685 n s$ \\
\hline Seguiu as instruções da bula vs período & $0,9617 n s$ \\
\hline Última consulta médica $v s$ período & $0,7001 n s$ \\
\hline Reaproveita medicamentos usados em tratamentos anteriores $v s$ período & $0,3471 \mathrm{~ns}$ \\
\hline
\end{tabular}

${ }^{n s}$ Não significativo ao nível nominal de $5 \%$ de significância $(p>0,05)$

${ }^{* *}$ Significativo ao nível nominal de $1 \%$ de significância $(p<0,01)$

Fonte: Autores. 
Figura 1 - Finalidade e perfil de uso dos medicamentos e condições de saúde na amostra de 110 alunos do curso de enfermagem de uma Universidade privada no Sul de Minas Gerais, 2018.

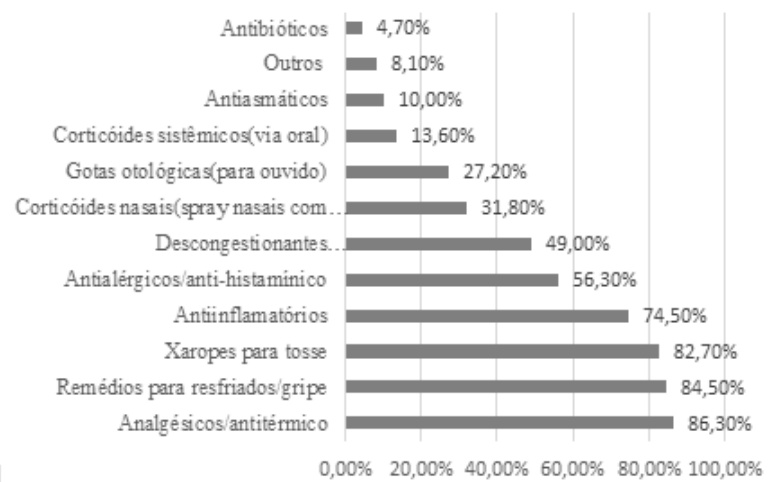

A. Medicamentos utilizados pelos acadêmicos

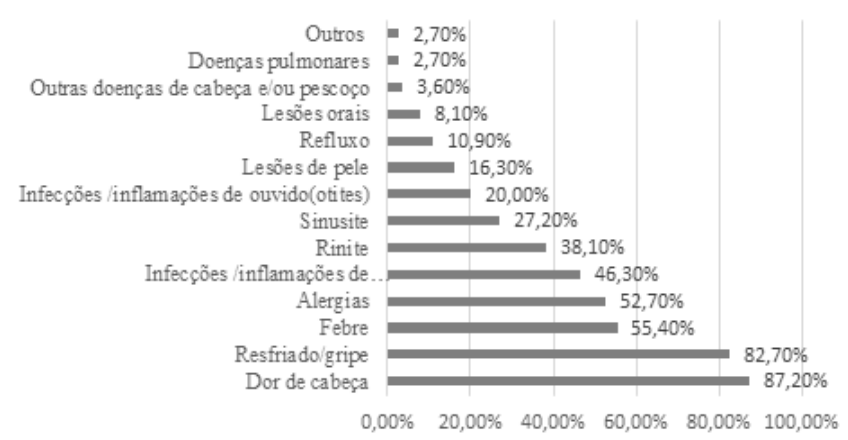

B. Motivos (patologias) que levaram os acadêmicos a automedicação

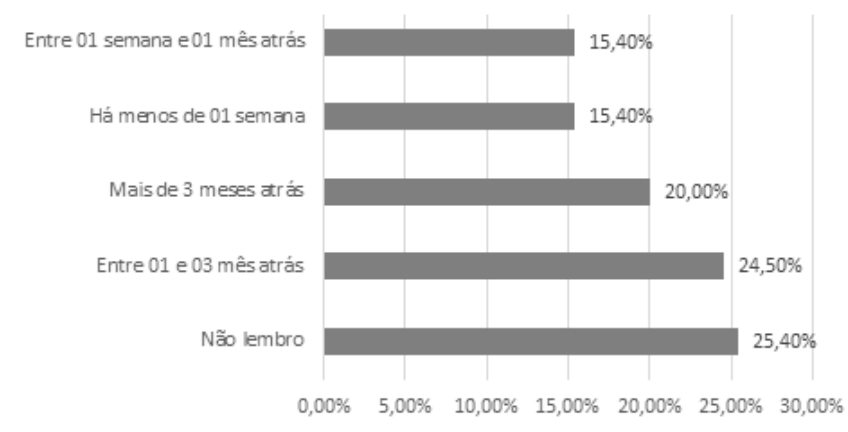

C. Periodo de realização da última consulta médica

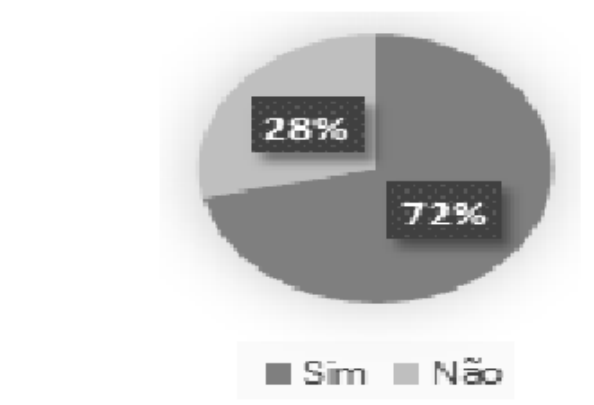

D. Reaproveitamento de medicamentos usados em tratamentos anteriores

Fonte: Autores.

\section{Discussão}

No presente estudo, uma amostra de 110 estudantes de enfermagem foi analisada, quanto ao uso de medicamentos. Houve predominância do sexo feminino e faixa etária entre 20-30 anos. Na área de saúde, a enfermagem é uma profissão singular, sendo numericamente uma prática quase exclusivamente feminina (Bandeira et al., 1998), o que explica a alta frequência de entrevistadas no presente estudo.

Em relação à automedicação, 94,5\% dos entrevistados já realizaram essa prática em algum momento da vida. Segundo Penna et al. (2004), em um estudo realizado com universitários no ano de 2004, este percentual variou entre 75,5\% e 92,0\%. Como levantado por Penna et al. (2004), os conhecimentos advindos da formação na área de saúde, poderia estar influenciando na alta frequência da prática de automedicação entre acadêmicos. Valores significativos também foram observados por Machado et al. (2020) ao apontar que, entre profissionais da área de enfermagem, a prática de automedicação foi entre 30$36 \%$, sendo que os profissionais atribuíram esta prática a falta de tempo para ir ao médico por conta da carga de trabalho excessiva. Os valores encontrados no presente estudo e por Penna et al. (2004); Machado et al. (2020) estão bem acima da frequência de automedicação em outros grupos populacionais, tais como os idosos, sendo que Bortolon et al. (2008) relataram que $26 \%$ entre todas as idosas atendidas no ambulatório de uma universidade em Brasília e inclusas no estudo praticavam automedicação. De acordo com Domingues et al. (2017), em um estudo envolvendo a população geral adulta de Brasília (2051 pessoas entre 18-65 anos), 14,9\% entre os entrevistados que usavam medicação praticavam automedicação, sendo que esta prática foi mais comum entre adultos jovens (18-34 anos) e menos comum entre idosos (50-65 anos). A promoção do uso racional de medicamentos pode contribuir para a diminuição do número de casos de intoxicação e internações hospitalares e, 
consequentemente, intensificar níveis de prevenção e promoção da saúde, permitindo melhor alocação dos recursos disponíveis. Segundo a com as diretrizes da Política Nacional de Medicamentos, deve-se dar ênfase ao processo de educação em saúde dos usuários ou consumidores de medicamentos, alertando eles sobre os riscos da automedicação, da interrupção e da troca da medicação prescrita, bem como sobre a necessidade de prescrição médica para aquisição de medicamentos (Brasil, 1998). Para tanto, é essencial o conhecimento dos hábitos atuais e tendências que levam certos grupos populacionais a praticarem automedicação, direcionando assim mais efetivas estratégias de prevenção.

Embora a maioria dos medicamentos utilizados pelos estudantes sejam isentos de prescrição (MIP), como levantado no presente estudo, 62,7\%, o consumo irracional pode gerar efeitos adversos e comprometer a saúde e, até mesmo, gerar óbitos. Além disso, o tratamento paliativo da dor pode dificultar ou impedir a elucidação do diagnóstico definitivo de doença e o tratamento adequado, contribuindo para a cronificação do processo bem como da experiência dolorosa.

O conceito OMS de saúde ou ausência de doença, que reflete o completo "bem-estar" físico, mental e social, sendo amplo, permite a experiência "pessoal" do enfrentamento ao problema de saúde, ou seja, personalizando o significado que o indivíduo confere a doença ou ao problema de saúde enfrentado. Portanto, a experiência de estar doente é única para o indivíduo que não é acompanhado de maneira adequada por um clínico, sendo os comportamentos adotados em situação de doença singulares e são influenciados por crenças e conceitos criados pela própria vivência e sociedade, dificultando o tratamento adequado e incentivando a automedicação (Graham et al., 2005). Ainda, os meios de comunicação incentivam a automedicação, comprometendo a saúde pública no Brasil (Nascimento, 2009; Lyra Jr. et al., 2010; Alhaddad, 2018; Azevedo et al., 2019; Gimenes et al., 2019). No presente estudo, apenas uma pequena parcela relatou apresentar problemas de saúde, e grande parte relatou reaproveitar receitas e medicamentos de tratamentos anteriores. Entre as queixas que levaram a aquisição de medicamentos, dor de cabeça (Cefaleia) é uma queixa frequente entre jovens e estudantes, assim como também observado no presente estudo, sendo esta, de fato, uma condição limitante gerando incapacidade temporária, déficit educacional e ausência escolar, causando prejuízo na qualidade de vida, sendo mais comum em mulheres e adultos jovens (assim como tipificado na amostra do presente estudo) (Curry \& Green, 2007). A alta frequência de relato desta queixa também justifica a maior frequência de uso da classe dos analgésicos, para alívio da dor. Resfriado e gripes foram também citados com frequência, justificando o segundo maior consumo de medicamentos, de antigripais e medicamentos para alívio do resfriado. Condições inflamatórias e uso de anti-inflamatórios foram também frequentemente citados. Quanto aos medicamentos mais comumente obtidos para automedicação, resultados semelhantes foram constatados por Bortolon et al. (2008); Machado et al. (2020), que mostrou maior consumo de anti-inflamatórios, analgésicos, antipiréticos e antialérgicos, além de medicamentos caseiros e fitoterápicos. Domingues et al. (2017) mostraram que analgésicos são também os medicamentos mais utilizados por automedicação entre a população geral de Brasília, mas que outras classes terapêuticas são também observadas entre a população geral analisada.

Por fim, a automedicação deve ser avaliada (WHO, 1998; TIPF, 1999), no contexto do uso racional de medicamentos (WHO, 2002), assim deve ser promovida uma conscientização entre a população sobre os efeitos deste hábito, além da necessidade de uma maior regulação pelos órgãos competentes sobre a veiculação de informações e propagandas em veículos de comunicação. Ainda, sempre é necessário um diagnóstico clínico para a instituição da terapia medicamentosa adequada (TIPF, 1999), com alternativas terapêuticas seguras e eficazes em doses, posologias e tempos de tratamento adequados para maior efetividade e segurança (WHO, 2002). De relevância clínica crítica, a automedicação não deve ser adotada por pacientes especiais, grupo que inclui gestantes, as crianças menores de dois anos, os idosos e os pacientes polimedicados ou com doenças severas e limitantes para medicação (TIPF, 1999). 


\section{Conclusão}

Houve predominância de acadêmicas frequentando o curso de enfermagem na instituição analisada, na faixa etária entre 20-30 anos, e com alta frequência da prática de automedicação, sendo este hábito mais comum entre os alunos dos períodos mais adiantados do curso. O reaproveitamento de receitas e medicamentos de tratamentos anteriores foi também comum. As automedicações para tratar dor de cabeça e gripes e resfriados foram as mais comuns, sendo estas condições também as mais relatadas. Portanto, destaca-se a necessidade na definição de estratégias voltadas à promoção do uso racional de medicamentos entre acadêmicos, principalmente visando reduzir os riscos de automedicação.

\section{Referências}

Alhaddad, M. S. (2018). The use of social media among Saudi residents for medicines related information. Saudi Pharmaceutical Journal, 26(8), 1106-1111.

Azevedo, J. M. B. J. M., et al. (2019). O marketing farmacêutico e sua influência no consumo de medicamentos: Uma revisão integrativa da literatura. Brazilian Journal of Health and Pharmacy, 1(4), 46-55.

Bandeira, L., et al. (1998). Representações de gênero e moralidade na prática profissional da enfermagem. Revista Brasileira de Enfermagem, 51(4), 677-696.

Bortolon, P. C., et al. (2008). Análise do perfil de automedicação em mulheres idosas brasileiras. Ciência \& Saúde Coletiva, 13(4), $1219-1226$.

Brasil. Ministério da Saúde. Aprova a Política Nacional de Medicamentos. Portaria GM no 3.916, 30 de outubro de 1998a. Lex: Diário Oficial da União, Brasília, 10 de nov. 1998.

Curry, K., \& Green, R. (2007). Prevalence and management of headache in a university undergraduate population. J Am Acad Nurse Pract, 19(7), 378-82.

Daniely, M. L., et al. (2017). Avaliação da prática da automedicação em acadêmicos do curso de farmácia em uma instituição privada de ensino superior em Fortaleza-CE. Revista Expressão Católica Saúde, 2(1).

Domingues, P. H. F., et al. (2017). Prevalence and associated factors of self-medication in adults living in the Federal District, Brazil: a crosssectional, population-based study. Epidemiol Serv Saude, 26(2), 12.

Freitas, N. A., \& Melo, O. F. (2018). Análise da automedicação por clientes em uma farmácia comunitária. Revista de Cultura, Ciência e Tecnologia Essentia (Sobral), 19(1), 31-39.

Gimenes, L. S., et al. (2019). A influência da propaganda de medicamentos na automedicação. Revista Amazônia: Science \& Health, 7(2), 14-19.

Graham, I. W., et al. (2005). Mutual suffering: A nurse's story of caring for the living as they are dying. International Journal of Nursing Practice, 11(6), 277285 .

Lyra Jr., D. P., et al. (2010). Influência da propaganda na utilização de medicamentos em um grupo de idosos atendidos em uma unidade básica de saúde em Aracaju (SE, Brasil). Ciência \& Saúde Coletiva, 15(Supl. 3), 3497-3505.

Machado, J., et al. (2020). Concepções sobre automedicação entre profissionais de enfermagem. Revista Brasileira de Pesquisa em Ciências da Saúde, 7(13), $10-15$.

Nascimento, A. C. (2009). Medication advertising in Brazil. Can it be regulated? Ciência \& Saúde Coletiva, 14(3), 869-877.

Organización Mundial de la Salud. Promoción del uso racional de medicamentos: componentes centrales. Genebra: Organización Mundial de la Salud [internet document] 2002.

Penna, A. B., et al. Análise da Prática da Automedicação em Universitários do Campus Magnus - Unipac Barbacena, MG. In: Anais do ${ }^{\circ}$ Congresso Brasileiro de Extensão Universitária.

Pereira, J. R., et al. (). Riscos da automedicação: tratando o problema com conhecimento. Universidade da Região de Joinville - UNIVILLE Pró-Reitoria de Extensão e Assuntos Comunitários - PROEX Área de Extensão Universitária, 20p., 2008.

Sousa, H. W., et al. (2008). A importância do profissional farmacêutico no combate à automedicação no Brasil. Revista Eletrônica de Farmácia, 1, 67-72.

The International Pharmaceutical Federation (TIPF), The World Self-Medication Industry. Responsible Self-Medication [Internet]. 1999.

Vilarino, J. F., et al. (1998). Perfil da automedicação em município do Sul do Brasil. Rev Saúde Pública, 32(1), 43-49.

World Health Organization. The role of the pharmacist in self-care and self-medication. World Health Organization; 1998. http://apps.who.int/medicinedocs/pdf/whozip32e/whozip32e.pdf 